\title{
CÁDIZ Y EL INTENTO AUTONOMISTA ANDALUZ DURANTE LA SEGUNDA REPUBLICA.
}

\author{
Manuel Hijano del Río \\ (Universidad de Málaga) \\ Manuel Ruiz Romero \\ (Universidad de Sevilla)
}

\section{$\underline{\text { RESUMEN }}$}

Estudio del impacto del movimiento autonomista andaluz en la ciudad de Cádiz durante los años de la Segunda República.

Palabras clave: España, siglo XX. Nacionalismo andaluz. Cádiz

\section{$\underline{\text { SUMMARY }}$}

Analysis of the reflect in Cadix of the autonomist andalousian movement during the years of the II Republic.

Cadix.

Keywords: Spain, XXth century. Andalousian nationalism.

No estaba suficientemente investigado el papel jugado por los municipios gaditanos en el intento de todos los andaluces por conseguir su autonomía durante la Segunda República, y más concretamente, en la celebración de la Asamblea Regional Andaluza de Córdoba de 1933. Faltaba una revisión de diversas fuentes documentales fundamentales como la prensa o de archivos importantes, como el de la Diputación, con objeto de analizar los pormenores de ese período histórico que consideramos tan importante, como poco conocido.

La Asamblea Regional Andaluza se celebra en Córdoba durante los días 29 al 31 de enero de 1933. Con esa reunión se culmina un proceso iniciado dos años antes, jalonado por multitud de abatares: sesiones dedicadas a este asunto de las instituciones provinciales y locales, izados de banderas andaluzas, llamamientos a la participación y la no participación...

Efectivamente, ya el 7 de mayo de 1.931 los nacionalistas, con Blas Infante a la cabeza, encuadrados en el seno del Partido Republicano Federal como Agrupación Republicana Federal Andaluza (A.R.F.A.), hacen un llamamiento de la Diputación hispalense para que convocase una Asamblea de organismos provinciales y elaborar un Estatuto de autonomía :

"Llegaron los tiempos que hubimos de predecir, desde hace veinte años, durante los cuales hemos venido exhortando al Pueblo Andaluz a que 
restaure su personalidad, la cual, afirmábamos entonces, tendrá que llegar a recobrar incluso, a causa de la exclusión determinada por las afirmaciones autonómicas de los demás pueblos peninsulares"².

El 13 de junio la Comisión Gestora de la Diputación de Sevilla acepta la propuesta y convoca una reunión con todos los presidentes de Diputación andaluces. El 26 de junio, la institución provincial gaditana contesta la primera de las ocho, por "la importancia que ha de tener la Asamblea que ha de celebrarse", señalando su Presidente, sin consultar con el resto de compañeros, que "me propongo por mi parte concurrir a ella"

En la sesión del 1 de julio de 1.931, la Diputación de Cádiz aborda la comunicación de su homónima hispalense ${ }^{4}$. El pleno designa al Presidente, Pedro Icardi Blanca, quien se compromete a que "antes de adoptar ninguna determinación, dará cuenta a esta Comisión gestora"

La reunión se celebra el 6 julio, tal como estaba previsto, y en la misma se acuerda enviar un cuestionario a todos los municipios de la región para que expusiesen su parecer respecto del propósito de las Diputaciones" ${ }^{\prime 6}$. Así mismo, se delega en una ponencia formada por los representantes de Cádiz, Jaén y Málaga para redactar un dictamen como base a la discusión. Estamos ante la primera consulta a los Ayuntamientos y entidades de Andalucía ${ }^{7}$.

Fruto de estas consultas iniciales, surge el "Proyecto de Estatuto del Gobierno Autónomo de Andalucía", elaborado entre el 11 de julio y el 3 de agosto de 1.931 por "personas competentes" designadas por el Presidente de la Diputación Provincial de Sevilla, Hermenegildo Casas, con la finalidad de "ganar tiempo y facilitar el trabajo"'. La ponencia remite copia del mismo a las Diputaciones, municipios y entidades, y a los diputados andaluces en el Parlamento, quienes en su mayor parte aconsejaron su paralización hasta que no fuese aprobada la Constitución republicana, argumento que condiciona los inicios de este intento autonómico ${ }^{10}$.

Este proyecto es el primer resultado concreto dado en Andalucía en favor de la consecución de su autonomía, tan sólo a unos meses tras la proclamación de la II República. Su contenido se mueve fuera del marco constitucional y ausente de un debate y de una conciencia general. Un texto abstracto y jurídicamente débil. Por ello, el documento no tuvo demasiadas repercusiones, salvo un primer pretexto para provocar un debate social en torno a la autonomía andaluza.

Mientras tanto, también el Ayuntamiento de Cádiz en su sesión del 4 de agosto de 1.931, faculta al Alcalde para que designe una ponencia que trate del problema autonómico, tal como se le solicitó desde la Diputación de Sevilla ${ }^{11}$. Un día antes, la Junta Provincial de Ganaderos de Cádiz estima que la autonomía "acaerraría los mayores males al interés nacional", aunque si bien el centralismo absorbente impide el desarrollo de las 
riquezas. ${ }^{12}$. El 14 de septiembre opina el diputado a Cortes por Cádiz, Antonio Roma Rubies promete estudiar el borrador y se ofrece para cuanto se le reclame ${ }^{13}$.

Aprobado ya el marco constitucional, en diciembre de 1.931, el 26 de febrero de 1.932 se reunen los representantes provinciales en Sevilla. A la reunión asisten todas las Diputaciones excepto Granada y Almería que se adhieren a los acuerdos que se adoptan ${ }^{14}$. En ella se distribuyen responsabilidades entre las corporaciones provinciales para la celebración de la Asamblea Regional que se prevee para el próximo mes de abril ${ }^{15}$. Todas las Diputaciones se ocuparían de elaborar listas de invitados y serían las responsables de las labores de información y difusión de la campaña. A la Diputación de Córdoba, además, le corresponderían las labores propias para la celebración del encuentro, creemos que por la situación geográfica de la ciudad califal ${ }^{16}$. La de Sevilla recibe la responsabilidad de ser la coordinadora general (Comisión Organizadora de la Asamblea Regional Andaluza) de las tareas administrativas a tenor de las informaciones enviadas desde las respectivas instituciones provinciales. Aquí se elaboran las Bases para un Estatuto de Andalucía con el fin de ser ponencia base a enmendar en la futura Asamblea de Córdoba ${ }^{17}$. Este proyecto, de corte moderado, es una "declaración de intenciones", que se ciñe a lo estipulado en el Título primero de la Constitución. Andalucía queda configurada en una simple "Mancomunidad de Diputaciones", en un texto preocupado excesivamente por priorizar el papel político de las Diputaciones sobre el de los municipios, que no aporta ningún avance cualitativo descentralizador, en clara continuidad con proyectos teorizados durante la Dictadura de Primo de Rivera. Tras su impresión por J. Mejías en Sevilla, se envía a los municipios andaluces en solicitud de pronunciamiento y enmienda.

A partir del 16 de marzo se suceden los aplazamientos. Ese día, José Andrés Vázquez, como Secretario de la Comisión Organizadora, anuncia que se celebrará entre los días 8 y 15 de mayo "por ser la época más propicia en Córdoba, para unos actos de la naturaleza e importancia de lo proyectado"18.

Conocido ya este primer aplazamiento, y viendo más fiable esa fecha, Cádiz convoca su reunión preparatoria para el 21 de abril. Unos días antes, Miguel Maldonado Pérez, en la Diputación, expone su malestar porque en el Proyecto de las Diputaciones aparezca que fue aprobado por las "diputaciones andaluzas", puesto que "si bien fue el acuerdo de la mayoría de los asistentes a la reunión previa en Sevilla, no lo ha sido por la Diputación de Cádiz, toda vez que él no fue facultado para ello, y sí lo hizo constar en aquel acto" ${ }^{19}$.

El Ayuntamiento de Jerez recibe, por otro lado, desde la Comisión Organizadora de la Asamblea Regional, un ejemplar de Estatuto el 16 de abril de $1.932^{20}$. En pleno, el concejal Mendoza advierte que este asunto ha 
de tratarse "sin premura", y se pregunta si la autonomía es sentida por el pueblo andaluz, o es el deseo de "imitar a otras regiones" o hay "otros fines particulares". También se acuerda que el Alcalde acuda a la reunión preparatoria provincial.

En la reunión preparatoria gaditana se dan cita un escaso número de representantes municipales, la mayoría provenientes del Campo de Gibraltar -Cádiz, Jerez, Algeciras, Grazalema, Chiclana, San Fernando y Puerto de Santa María-, que se contrarresta con la elevada presencia de entidades, como la Sociedad Económica de Amigos del País de Cádiz, el Ateneo Jerezano, el Colegio Oficial del Secretariado local, la Academia de Bellas Artes, el Instituto de Segunda Enseñanza, el Colegio Oficial de Interventores, así como la importante presencia de Diputados a Cortes: Fermín Aranda (republicano), Manuel Moreno Mendoza (republicano radical), Manuel Muñoz Martínez (radical socialista), y Francisco Aramburu e Inda (republicano derechista) ${ }^{22}$, quienes además contaban con la representación de Emilio de Sola (republicano), y Santiago Rodríguez Piñero (republicano) ${ }^{23}$. También asisten el Presidente de la Diputación, Pedro Icardi, y los vocales de la Comisión Gestora de la Diputación, Dorado, Maldonado y Campuzano, quien, a su vez, ostenta la representación de Antonio Butrón. Tenemos constancia de la asistencia de representantes de la Asociación Gremial portuense, la Cámara Industrial de Comercio y Navegación de Algeciras, el Colegio Oficial de Interventores, la Unión Patronal Gaditana, el Ateneo de Cádiz, la Agrupación de la Prensa, el Centro Mercantil e Industrial, la Cámara de Comercio, Industria y Navegación de Cádiz y la Escuela Profesional de Artes y Oficios ${ }^{24}$.

En el salón de la Diputación Provincial se recaba la opinión de la provincia:

- el grupo de los contrarios a la autonomía, formado, casi exclusivamente por Carlos Derqui, de la Unión Patronal Gaditana, el primero en intervenir en la Asamblea. Ellos identifican la autonomía con independencia, y además, añaden la falta de conciencia de los andaluces para aprobar un Estatuto:

"Pero, emanciparnos del Estado nacional, para echarnos en brazos de un Estado regional, con todos los mayores respetos, para las personas que substenten esta idea, pero con ninguno para la idea, es dar vida al dicho vulgar de "salir de Málaga para entrar en Malagón""25.

- el grupo de representantes decididamente a favor de la autonomía, con Mariano López Muñoz, interventor del Ayuntamiento del Puerto de Santa María, a la cabeza, y apoyado por el Alcalde de Chiclana, Javier Cruz Cortijo, que reconocen traer un mandato: "la defensa de la autonomía y en sostener y votar la Diputación regional única, en la que cristalice la voluntad de los Ayuntamientos" 
- un tercer grupo, formado especialmente por todos los diputados a Cortes que toman la palabra en la Asamblea -Muñoz Martínez, Moreno Mendoza y Fermín Aranda-, que se consideran favorables a la autonomía, pero creen necesario, primero, una labor de concienciación o, en otros casos, se duda de la representatividad de los allí reunidos. Así, Moreno Mendoza, en su primera intervención dice:

"no despreciamos la idea, pero no tenemos la representación que se desea para que todos podamos decir que vamos en representación de una provincia que está al tanto de todo con perfecto conocimiento (...) Expresa que España no está reconstituida todavía: tejemos de una manera distinta a como estaba constituida, y por tanto, se debe esperar y estudiar, porque no estamos en el caso de la región catalana" ${ }^{\prime 27}$.

Muñoz Martínez, añade: "por su credo político que admite lo que en en la Constitución está estatuido (sic), y además porque considera como andaluz que nuestra región tiene fuentes de riqueza política, social y económica", pero "mientras que no se haga una educación, una vulgarización de la política regional, el pueblo no podrá emitir su opinión. Habla del problema de Cataluña y de que si se hiciera como allí un plebiscito, el pueblo, no preparado, se preguntaría: ¿qué hacemos?"28.

- Por último, cabe distinguir otro grupo de representantes que consideran no estar capacitados para emitir una opinión sobre el tema, o la falta de representatividad del encuentro.' Así, sólo se expresa el Alcalde de Cádiz y "por alusiones", Enrique Alvarez López. El silencio del resto de representantes municipales -Jerez, Algeciras, Grazalema- y de las entidades particulares, confirma su adscripción a este grupo de observadores.

Al final, la opinión de los diputados es la que se impone entre los asistentes y es asumida como acuerdo. Consideran asistir a Córdoba, pero además, hacer una campaña de difusión de la autonomía.

La celebración del foro preparatorio consigue llevar la reflexión a la prensa gaditana, la cual se convierte en una rica profusión de opiniones a favor y en contra de la autonomía. Las opiniones contrarias al Estatuto -la mayoría- se basan en argumentos tales como la españolidad de Andalucía y el centralismo sevillano:

"¿Qué se pretende?, ¿imitar a Cataluña?, ¿seguir los pasos de Vasconia?, ¿plagiar a los gallegos?, ¿Andalucía, región emintentemente española, que nunca, nunca ha pensado en romper la unidad de la patria va ahora a pedir descentralizarse? (...) ¡una Mancomunidad de Diputaciones Provinciales, con vistas a centralizarla en una sola provincia de las que hoy forman el conglomerado andaluz!" ${ }^{29}$.

Tampoco faltan las críticas al procedimiento seguido, en el que sólo han participado las Diputaciones y, por lo tanto, sin tener en cuenta la 
opinión del pueblo, además de alusiones a la falta de conciencia autonómica andaluza ${ }^{30}$.

"Ya se sabe que no se trata de una Andalucía separatista, ni siquiera de alcanzar una autonomía política, sino exclusivamente de una seudo autonomía administrativa, pero es que aún así al andaluz no le interesa. Por instinto advierte que si el cacique andaluz dispone además de los resortes tributarios, teniéndolos al lado de casa, conociendo hasta en sus mínimos detalles las posibilidades de su vida económica, su vida se la dificultará. (...) Andalucía (...) no debe jugar con el vocablo Estatuto" ${ }^{31}$.

Las opiniones favorables, parten casi siempre de los círculos nacionalistas, como Estanislao Sánchez López, Secretario del Ayuntamiento de Arcos de la Frontera:

"debe llevarse a cabo la redacción de un proyecto más completo aportando los materiales que indican los intereses verdaderamente peculiares de Andalucía, y que debe concretarse en una ponencia que redacten personas técnicas, para que difundida profusamente entre todos los ciudadanos y organismos de la región, puedan proponer las adiciones o modificaciones que respondan a la realidad" $"$.

Este representante municipal, que asiste a la reunión preparatoria, hace la siguiente valoración: "fue apreciado que con el actual proyecto no pueden adquirirse compromisos para la provincia, porque no encaja en la órbita constitucional. Reconociéndose además que no se ha hecho la propaganda que corresponde a la magnitud del problema" ${ }^{33}$.

Tomás Orellana Massa, "ilustre decano de la Beneficiencia de Sevilla", manifiesta que la Diputación Provincial de la ciudad hispalense ha tenido "un máximo acierto" al iniciar la formación de la mancomunidad, "y a ella debemos ir todos no por pasión, sino por reflexión", reconoce que Andalucía es una "región natural", cuya base fundamental "es el enlace de los problemas" ${ }^{34}$.

La actitud de otros era expectante, como la del Presidente del Ateneo Jerezano, Tomás García Figueras:

"Yo comparto el patriótico temor y el justo recelo de los que, a primera vista, han podido ver en esta cuestión un fondo político, un deseo de autonomía imitador de malsanas tendencias (...). Pero téngase en cuenta que ésta fue la primera corriente de opinión que recogieron los iniciadores del Estatuto (...) creo que debe irse a escuchar, (a Córdoba), a intervenir si es necesario, a pulsar de cerca el espíritu que anima a los allí reunidos" ${ }^{35}$.

Días después de la reunión preparatoria gaditana ${ }^{36}$, la Comisión de Gobernación del Ayuntamiento de Cádiz manifiesta haber recibido el Estatuto de las diputaciones, y por la proximidad de la nueva fecha de la 
Asamblea Regional, actúa rápidamente: designa al Alcalde como representante y se nombra una ponencia formada por los miembros de los partidos, que estudie el Estatuto. A pesar de que el debate se restringe: a la comisión nombrada, algunos concejales toman la palabra para emitir su opinión. La intervención más destacada es la del Alcalde, que informa al pleno de la reunión preparatoria, celebrada en un "ambiente de cordialidad" y "extraordinaria mesura" ${ }^{37}$, adoptándose el acuerdo de divulgar entre el pueblo la idea del regionalismo.

El Alcalde, en calidad de presidente de la comisión designada para esta cuestión lee, el 30 de abril, las conclusiones de las deliberaciones. Éstas muestran su desacuerdo con el sistema seguido para la redacción del proyecto y para convocar la Asamblea Andaluza: "dicho proyecto no ha partido ni del mưnicipió ni menos aún del cuerpo electoral" ${ }^{38}$. Dudan de la conciencia regional del pueblo y de lo impreciso del proyecto, sobre todo en temas municipales: "haciendo caso omiso de los municipios, y como es natural, éstos al ignorar los derechos que se le reservan, no pueden en modo alguno hacerse solidarios de un Estatuto de tal forma concebido" ${ }^{39}$. Por último, la ausencia de una Ley Provincial que defina el papel de las Diputaciones hace del proyecto incongruente, puesto que organiza la región "asentándose en las provincias". El Alcalde señala el peligro de convertir la autonomía en una "máquina burocrática más, un tanto mohosa":. Las conclusiones son asumidas por el pleno, y el Alcalde manifiesta su intención de asistir a Córdoba.

El representante jerezano informa a su Ayuntamiento de la reunión preparatoria. El Alcalde, Francisco Lerma Alsina, manifiesta la escasa asistencia de los ayuntamientos, aunque se acordó acudir a Córdoba, "sin votos particulares" ${ }^{41}$. Los concejales intervienen para mostrarse contrarios a asistir a Córdoba. En este sentido se expresan Santiago Lozano Carralón, Juan Taboada Jiménez, y Vicente Castilla Flores. Confiados en que se podrá estudiar lo que allí se apruebe, y así también evitar compromisos, 26 ediles acuerdan no asistir. Sólo tres, Isidro González González, Enrique Rubio Morales y José Almagro Casas, se manifiestan en contra. Sin embargo, el Ayuntamiento, más adelante, cambia de opinión: envía al Teniente Alcalde Victoriano Romero Palomo ${ }^{42}$.

La Diputación ante el anuncio de que la Asamblea de Córdoba será entre el 8 y el 15 de mayo, celebra una sesión el 27 de abril, donde se designa a Pedro Icardi, Juan Antonio Campuzano y Miguel Maldonado como representantes. Éstos ya habían asistido a la reunión preparatoria, de la que dicen "reinó un espíritu opuesto a que se adopten resoluciones de carácter decisivo, puesto que no se ha oido la voluntad del pueblo de tan excepcional importancia ${ }^{\text {"43 }}$.

Sin embargo, a pesar de todos estos preparativos, el 2 de mayo se aplaza de nuevo la fecha de la Asamblea Regional, justificando este nuevo retraso al volumen de trabajo que en estos días tienen los diputados a 
Cortes con la discusión del Estatuto autonómico catalán: "'un numeroso grupo de Diputados a Cortes por las ocho provincias andaluzas' ha rogado el aplazamiento, pues 'les impediría asistir a la discusión del estatuto el supremo interés del trabajo parlamentario que en las mismas fechas habrá que desarrollarse en la Cámara"' ${ }^{44}$.

El inicio del debate catalán el 6 de mayo propicia unas movilizaciones y protestas sobre los contenidos y métodos de la autonomía para Cataluña. Cuestión aprovechada por los elementos reaccionarios, contrarios a la República para cuestionar la confianza y la viabilidad del régimen y su orden constitucional vigente. No cabe duda que el debate social creado en este primer debate parlamentario sobre la autonomía en Cortes afecta sustancialmente al impulso autonomista andaluz, inscrito éste en un contexto social poco sensible. Es posïble, por tanto, que Ta Cumisión Organizadora a la vista del estado de opinión creado durante los cinco meses del debate catalán (mayo-septiembre) abogara por una prudente e intencionada ralentización del intento autonómico. Lo que significa un abierto cambio de ritmo en Andalucía con respecto a los primeros meses de la República.

La desmotivación y el desconcierto tuvieron que ser patentes entre las fuerzas políticas y sociales gaditanas debido a estos aplazamientos. Los esfuerzos gastados en horas de reunión se veían frustrados por estos retrasos que, a su vez, dejaban entrever una gran desorganización y falta de apoyo institucional (diputados a Cortes, fuerzas políticas, otras Diputaciones, ...); razones que sin duda alentaba los argumentos contrarios a la autonomía.

El 20 de octubre la Comisión vuelve a anunciar que la Asamblea "se celebrará en Córdoba, el próximo mes de noviembre en fecha no determinada aún, y que se comunicará a su debido tiempo" ${ }^{45}$. Finalmente, el inicio de la campaña de difusión de los símbolos andaluces, la campaña al Parlamento catalán, el XIII Congreso del PSOE, la ausencia previsible de los parlamentarios en Cortes, la definitiva editorial de "El Socialista", la opinión pública, ... recomendarían su celebración. Finalmente, la Comisión Organizadora fija las fechas del 29 al 31 de enero.

A partir de aquí, diversos actos se suceden los días previos a la Asamblea de Córdoba. Blas Infante pronuncia una conferencia en Jerez, sobre el Estatuto en octubre de 1.932, de la que se concluye la necesidad de la autonomía, como resultado de la crisis del Estado unitario ${ }^{46}$. En el mismo sentido, Ricardo Majó Puig (autonomista) acude el 30 de diciembre de 1.932 al Ateneo Jerezano, para dar una conferencia sobre "la autonomía andaluza en su aspecto económico". Explica el político autonomista que Andalucía posee una historia y una geografía diferentes al resto de España: "viendo la Federación española como un hecho biológico de devenir histórico". Propone una Hacienda regional con los impuestos directos, mientras que los indirectos serían para la Hacienda central. Pretende la 
asociación de patronos y obreros para lograr la "paz y la armonía entre las distintas clases sociales", y aboga por la inversión en la explotación de los recursos naturales andaluces ${ }^{47}$.

El 12 de noviembre de 1.932, el concejal del Ayuntamiento de Jerez, García Romero, propone que de acuerdo con "la autonomía que pueden disfrutar las Regiones dentro de lo que les autoriza la vigente Constitución, propone la celebración de un acto homenaje á la bandera regional izándola en la Casa Consistorial", demostrando de este modo, "que Jerez libre será andaluz y Andalucía libre será España"48. La propuesta es asumida por todas las fuerzas políticas, y se encarga al Archivero del Ayuntamiento para que prepare los detalles. Seis días más tarde, el Ayuntamiento de Jerez envía una carta inquiriendo al Presidente de la Diputación de Sevilla sobre "cuales son los auténticos colores y forma en que se distribuyen, de la bandera andaluza y de ser factible, qué concepto o hecho histórico funda su heráldica particular muy importante que evitaría la equivocación consiguiente, que de existir restaría grandeza al emblema que se trata de enaltecer" ${ }^{\prime 49}$. El Presidente responde al Secretario del Ayuntamiento al día siguiente indicándole las características de la bandera, añadiendo su historia en una nota adjunta ${ }^{50}$. Coinciden todos estos acontecimientos con la intensificación de la "propaganda de los andalucistas" en la provincia ${ }^{51}$.

El 8 de enero de 1.933, Jerez homenajea a la bandera andaluza, en un acto que cuenta con una asistencia "numerosa". En el mismo, el Alcalde señala que no es resultado de las gestiones en pro de la autonomía. Sólo se trata de ensalzar la enseña de Andalucía, puesto que hay que formar el "alma andaluza" para que se produzca la autonomía ${ }^{52}$. En primer lugar, se interpreta el Himno de la República, unas palabras del Alcalde "alusivas al acto" e iza la Bandera a los acordes del pasodoble "La Giralda", "composición adoptada como himno andaluz, y después el nacional". Finaliza el acto con un concierto de la Banda municipal ${ }^{53}$.

Días antes a la Asamblea de Córdoba, la Diputación de Cádiz recibe un llamamiento de la de Sevilla, tras el exitoso resultado de su reunión preparatoria: "fue acuerdo unánime dirigir efusivos saludos y expresarle deseo de que se cumpla propósito objeto Asamblea mediante máxima cooperación fraternal para bien de Andalucía, España y la República" ${ }^{54}$. La Diputación insiste en que sus representantes no pueden contraer compromiso alguno, al ser este organismo incapaz de resolver en una cuestión "tan importante y trascendente", puesto que "no son de elección popular"

La representación gaditana en la Asamblea Regional estaba compuesta por diez personas:

Diputación: Juan Antonio Campuzano y Miguel Maldonado Sierra.

Diputado a Cortes por la Línea de la Concepción: Adolfo Chacón Mata. 
Ayuntamiento de Grazalema: Manuel Rodríguez Matres

Ayuntamiento de Jerez: Victoriano Romero Palomo

Ayuntamiento del Puerto de Santa María: Mariano López Muñoz

Ayuntamiento de San Roque: Antonio Galiano y Leocadio Rico Campuzano.

Ayuntamiento de Sanlúcar de Barrameda: Manuel Ruiz Delgado y Guillermo Díez Cuevas.

Partido Radical Socialista: Manuel Rodríguez Matres

Junta Liberalista: Manuel Rodríguez Matres ${ }^{56}$.

Además de todos los ayuntamientos nombrados, tenemos datos que muestran las distintas posiciones de otros más como los de Alcalá de los Gazules, Arcos de la Frontera, Benaocaz, Chipiona, Chiclana, Conil, Grazalema, La Línea, Trebujena y Ubrique ${ }^{57}$.

En síntesis, habría que matizar las opiniones que otras investigaciones han caracterizado a la provincia de Cádiz como decididamente a favor de la autonomía. Diversos datos nos demuestran que el proceso autonómico no contó con apoyos tan decididos, como en un primer momento pudiera pensarse. Así, a la reunión preparatoria gaditana no asistieron un buen número de Ayuntamientos. Tan sólo, y tal como nos recuerda Pedro Icardi en la crónica de esa reunión, las localidades que configuran el entorno de la Bahía de Cádiz. El resto, deja en manos de entidades particulares o personajes la vida pública la decisión. No podemos achacar, como ocurrirá en Córdoba, a la falta de organización o a la desidia de la Diputación; la falta de los representantes municipales, puesto que desde el mes de julio de 1.931 , la institución provincial realiza una campaña de promoción de grandes magnitudes, comparables a provincias de claro talante autonomista. La resonancia de esta campaña también lleva a pensar que en Cádiz los planteamientos autonómicos cuentan con un gran apoyo. Sin embargo, y como hemos visto, esas acciones son tan sólo ejercicio de un "exceso" de celo de la Diputación gaditana, que no se vería correspondido en el resto de Andalucía, y ni mucho menos en la misma provincia.

La presencia en la provincia de destacados nacionalistas, como Antonio Chacón Ferral o Mariano López Muñoz, insinúa la posibilidad de que éstos lleven a cabo una intensa labor andalucista. No hemos encontrado nada que corrobore esa hipótesis. Las escasas apariciones en prensa son contrarrestadas con opiniones procedentes de ámbitos menos autonómicos y que llegan a calar más profundamente en pequeños sectores de la opinión pública gaditana. Cádiz no asiste a Córdoba con una ponencia común. Los Ayuntamientos asistentes y los representados son escasos, lo que contrasta con la fuerte presencia, y actuación, de los diputados a Cortes.

Mención especial merece el Ayuntamiento de Cádiz capital, con mayoría monárquica, que desempeña una labor indecisa que, finalmente, desemboca en la no asistencia a Córdoba. Este ejemplo, puede explicar la falta de muchos ayuntamientos gaditanos en Córdoba y en la preparatoria, 
ya que la mayoría de los concejales electos en abril de 1.931 pertenecen precisamente a las candidaturas monárquicas. Así, la implantación lograda por las fuerzas republicanas-socialistas en algunas localidades, por contra, explicaría actos puntuales como el izado de banderas andaluzas en Algeciras o Jerez ${ }^{38}$, en los prolegómenos de la Asamblea.

\section{NOTAS:}

1 INFANTE, B., La verdad sobre el Complot de Tablada y el Estado Libre de Andalucía, Granada,Aljibe, 1.979.

2 El Noticiero Sevillano, 13 de mayo de 1.931, p. 3. La solicitud está firmada por la Junta Liberalista de la Agrupación Republicana Federal, compuesta por Francisco Chico Ganga, Mariano López Muñoz, Rafael Ochoa Vila y Blas Infante.

3 DIAZ ARRIAZA, J., y RUIZ ROMERO, M., El proceso autonómico de Andalucía durante la II República, Sevilla, Fundación Blas Infante, 1.991, p. 43.

4 A.H.CA., Actas de la Diputación de Cádiz, sesión del 1 de julio de 1.931 , fols. $160-161$.

5 Id. Sin embargo, Pedro Icardi no pudo asistir y se designa a Juan Antonio Campuzano.

6 LACOMBA, J.A., Regionalismo y autonomía en la Andalucía contemporánea (1.835-1.936), Granada, Caja General de Ahorros de Granada, 1.988, p. 266. A la reunión asisten: Hermenegildo Casas, Camilo Chousa, José Guerra Lozano, Filiberto López, Juan Antonio Campuzano, Manuel Gracia Pérez, Estanislao del Campo, Manuel Olmedo Serrano, Luis Vargas Durán, Montes del Castillo y Cobos Estrada. DIAZ ARRIAZA, J., y RUIZ ROMERO, M., op.cit., p. 44.

7 La respuesta a esta primera consulta en Ibíd, pp. 48-63. Siendo el primer Ayuntamiento que responde a Marinaleda. Sólo se ha documentado las respuesta de los municipios de Cádiz y Sevilla. En otras provincias la prensa se encarga de informar.

8 A.D.P.S., Leg. 530, "Proyecto de Estatuto del Gobierno autónomo de Andalucía", Ejemplar mecanografiado.

9 DIAZ ARRIAZA, J., y RUIZ ROMERO, M., op.cit., p. 63 y p. 221, nota 351 . 
10 Recordemos que la Constitución de la Segunda República española no se aprobó hasta el 9 de diciembre de 1.931. El contenido de las respuestas hasta ahora localizadas de los diputados a Cortes, municipios y entidades a este proyecto se analizan en DIAZ ARRIAZA, J., y RUIZ ROMERO, M., op.cit., pp. 51 y ss.

11 A.M.CA., Actas del Ayuntamiento de Cádiz, sesión del 4 de agosto de 1.931, fols. 255-256.

12 DIAZ ARRIAZA, J., y RUIZ ROMERO, M., op.cit., p. 60.

13 IbId., p. 67.

14 LACOMBA, J.A., op.cit., p. 266.

15 En concreto, el orden del día de esta reunión fue el siguiente: 1) aprobación del citado Anteproyecto que se ha de someter a la próxima Asamblea; 2) señalar lugar y fecha de esa Asamblea; 3) amplitud que debe tener la invitación a la Asamblea regional; 4) propuestas de las Diputaciones provinciales para las invitaciones a los organismos y personalidades de sus respectivas provincias; 5) normas generales para la organización de la Asamblea. Id..

16 También creemos que se elije a Córdoba, por su situación geográfica con respecto a Madrid, para no obstaculizar la presencia de diputados en Cortes-; por ser un lugar histórico -capital del Califato-, a la par lo fue ya para la organización regional de Andalucía -Asamblea de 1.919, al margen de que fuera importante celebrarla en otra capital que no fuera Sevilla.

17 Estas Bases también se encuentran recogidas en LACOMBA, J.A., "Historia del Proyecto Andaluz de Estatuto de autonomía", en Gibralfaro, 28, (1.976), y en el Diario de Málaga, de 30 de enero de 1.933. La totalidad del Estatuto y la crónica del encuentro en El Liberal y El Noticiero Sevillano, 27 y 28 de febrero de 1.932. El texto se vuelve a repetir en El Liberal, 8 de abril de 1.932, p. 3.

18 DIAZ ARRIAZA, J., y RUTZ ROMERO, M., op.cit., p. 74. Efectivamente, para esos días se organizaron excursiones paralelas a la celebración de la Asamblea a la Sierra, la Mezquita, el Castillo de Almodóvar, Museos, el Pantano de Guadalmellato, ... LACOMBA, J.A., Regionalismo y ..., op.cit., p. 268.

19 A.H.CA., Actas de la Diputación de Cádiz, sesión del 6 de abril de 1.932 , fols. 101 y 102 . 
20 A.M.JE., Actas del Ayuntamiento de Jerez, sesión del 16 de abril de 1.932, fols. 92 y 93. Agradecemos a D. Cristóbal Orellana, Archivero Municipal de Jerez, las facilidades prestadas a los autores de este trabajo.

21 Estos tres diputados figuraron en las listas de la conjunción republicano-socialista. Merece destacarse a Manuel Muñoz, miembro del Partido Republicano Radical Socialista Independiente, comandante de Infantería retirado, y principal figura de los radicales-socialistas en la provincia. Es destacable la ausencia de los representantes socialistas.

22 Miembro de la Derecha Liberal Republicana, comerciante y miembro de una acreditada familia gaditana de banqueros.

23 Éstos dos últimos fueron miembros de la conjunción republicanasocialista por Cádiz en las elecciones a Cortes Constituyentes. Sola era, además, Presidente Provincial del Partido Republicano Autónomo de Cádiz.Sobre la filiación de los diputados a los partidos políticos gaditanos: CARO CANCELA, D., La Segunda República en Cádiz. Elecciones y partidos políticos, Cádiz, Diputación de Cádiz, 1.987, p. 110 .

"El Proyecto de Estatuto de Andalucía. La reunión de ayer en la Diputación", Diario de Cádiz, 22 de abril de 1.932, pp. 1y 4, "Reunión en la Diputación Provincial para tratar el Estatuto Andaluz", Diario de Jerez, 22 de abril de 1.932, p. 1 y "El Estatuto de autonomía. La reunión de ayer en la Diputación", Diario de Cádiz, 21 de abril de 1.932 , p. 3.

25 "El Proyecto de Estatuto de Andalucía. La reunión de ayer en la Diputación", Diario de Cádiz, 22 de abril de 1.932, pp. 1-2.

$\underline{\text { Id. }}$

27 Id.

Id..

El Guadalete, 23 de abril de 1.932, p. 1.

CHACON, M., "El Estatuto catalán, los estatutos regionales y el Estatuto andaluz", El Guadalete, 6 de mayo de 1.932, p.1 . Del regionalismo se dice en Cádiz: "el regionalismo es, ha dicho alguien que no me llega a la memoria, pero sí uno de sus más autorizados corifeos, es el preservativo contra el internacionalismo". "Regionalismo territorial y regionalismo político", Diario de Cádiz, 8 
de octubre de 1.932 , p. 1. Cuatro días más tarde, también se reproduce en la prensa cordobesa: "Regionalismo territorial y regionalismo político", El Sur, 21 de octubre de 1.932, p. 1.

32 Diario de Cádiz, 30 de abril de 1.932 y El Guadalete, 28 de abril de 1.932, p. 1 .

33 Id..

34 "Intereses regionales. Sobre la Mancomunidad andaluza en proyecto. Opinión del Doctor Orellana y Massa", Diario de Cádiz, 15 de abril de 1.932, p.1. Otros textos, también en el mismo sentido: "De regionalismo andaluz", Diario de Cádiz, 27 de abril de 1.932, p. 1, firmado por "Un regionalista; y HERCUES, "Andalucismo. Balance de 1.932", Diario de Cádiz, 10 de enero de 1.932.

35 El Guadalete, 4 de mayo de 1.932, p. 1.

36 A.M.CA., Actas del Ayuntamiento de Cádiz, sesión del 23 de abril de 1.932, fols. 192-194.

37 Id.

38 A.M.CA., Actas del Ayuntamiento de Cádiz, sesión del 30 de abril de 1.932, fols. 228-230. La Comisión estaba formada por el Alcalde, y los concejales Silvian, minoría radical; Hijano, socialista; Campos, radical-socialista; Gámez, Servicio de la República; además un representante de las minorías que se eligió entre Abella, de la Puente, Carrera y Llergui. Las conclusiones se encuentran también en el A.D.P.S., Leg. 530, Carta de firma ilegible al Presidente de la Comisión Organizadora de la Asamblea Regional.

39 A.M.CA., Actas del Ayuntamiento de Cádiz, sesión del 30 de abril de 1.932, fols. 228-230.

40 Id.

41 .M.JE., Actas del Ayuntamiento de Jerez, sesión del 27 de abril de 1.932, fols. 112-113.

42 A.M.JE., Actas del Ayuntamiento de Jerez, sesión del 28 de enero de 1.933, fol. 292.

43 A.H.CA., Actas de la Diputación de Cádiz, sesión del 27 de abril de 1.932, fols. 120 y 121. Los gastos ocasionados por la posterior visita de Juan Antonio Campuzano y Miguel Maldonado a Córdoba en enero de $1.933-903,8$ ptas.- fueron cargados al presupuesto de la Diputación de 
Cádiz, al capítulo de "representación provincial". A.H.CA., Actas de la Diputación de Cádiz, sesión del 25 de febrero de 1.933, fol. 40 vto..

${ }^{44}$ DIAZ ARRIAZA, J., y RUIZ ROMERO, M., op.cit., p. 86.

Ibíd., p. 76.

46 Claridad, 11 de octubre de 1.932 , p. 4.

47. El Guadalete, 31 de diciembre de 1.932 , p. 1. También aparece una reseña de esta conferencia en El Liberal, 4 de enero de 1.933 , p. 4. Parece ser que la conferencia no respondió a las expectativas suscitadas, ya que Tomás García, presidente del Ateneo Jerezano dijo ese día: "es de lamentar que el Sr. Majó no hubiere comenzado por el estudio del aspecto económico del Estatuto Andaluz, pues si bien es cierto que los antecedentes eran de interés habría tratado la cuestión con más detalle y hecho una labor más eficaz". Casas manifiesta el 21 de marzo de 1.932 al Presidente de la Diputación de Cádiz que recibe correspondencia del Ateneo Jerezano, "en la que se muestran muy interesados en la Asamblea. Como esta entidad pertenece a esa provincia traslado a Vd. ese interés por si cree conveniente incluir dicho Ateneo en la propuesta de invitaciones que esa Diputación de esa digna Presidencia habrá de remitirnos". A.D.P.S., Leg. 530, Carta del Casas al Ilmo. Presidente de la Diputación de Cádiz, de fecha 21 de marzo de 1.932 .

A.M.JE., Actas del Ayuntamiento de Jerez, sesión del 12 de noviembre de 1.932 , fol. 155 .

49 A.D.P.S., Leg. 550, Carta de la Alcaldía de Jerez al Presidente de la Diputación de Sevilla de fecha 18 de noviembre de 1.932. No es de extrañar esta pregunta, ya que durante 1.932 se izaron "banderas andaluzas" de la más variada composición. Así, tenemos constancia de que la bandera izada en Dos Hermanas, estando presente Blas Infante, se componía de las tres franjas verde, blanca y verde, pero con un sol rojo, "simbolizando al Imperio del Sol Poniente, sobre el cual y en plata, lleva el escudo de Cádiz", colocados, ambos, sobre la franja blanca. "Andalucismo. Balance de 1.932", Diario de Cádiz, 10 de enero de 1.932.

50 A.D.P.S., Leg. 550, Carta del Presidente de la Diputación de Sevilla al Secretario del Ayuntamiento de Jerez, de fecha 19 de noviembre de 1.932. Ese mismo día, Casas envía al Ayuntamiento jerezano un ejemplar de las Bases para el Proyecto de Estatuto, destinado a García Romero, quien el 8 de noviembre se lo había pedido "por ser muy probable que vaya a la Asamblea de Córdoba en representación del Ayuntamiento de esta población, (...) asunto con el que estoy 
verdaderamente encariñado". A.D.P.S., Leg. 550, Cartas del Concejal García Romero al Presidente de la Diputación de Sevilla y viceversa, de fechas 8 y 19 de noviembre de 1.932, respectivamente.

51 Un autonomista que firma bajo el seudónimo de HERCUES escribe en enero de 1.933: "Casi coincidiendo con el último trimestre, se acentúa la propaganda de los andalucistas" HERCUES, "Andalucismo. Balance de 1.932", Diario de Cádiz, 10 de enero de 1.932.

52 Al acto se invita a Hermenegildo Casas. Sin embargo, el Presidente de la Diputación sevillana excusa su asistencia: "Saludo felicito esa Corporación que confirma su recio abolengo andaluz". A.D.P.S., Leg. 550, Carta de la Alcaldía de Jerez al Presidente de la Diputación de Sevilla de fecha 24 de diciembre de 1.932 y Carta de Hermenegildo Casas al Alcalde de Jerez, sin fecha. Sobre este acto, además: Diario de Jerez, 7 de enero de 1.933, p.1 y Diario de Jerez, 10 de enero de 1.933, p. 1.

53 El Ayuntamiento de Jerez aprueba celebrar este acto en la sesión del 17 de diciembre de 1.932. Aunque en principio se acuerda el 1 de enero, sin embargo, debido al mal tiempo, el homenaje se pospone al día 8. A.M.JE., Actas del Ayuntamiento de Jerez, sesión del 17 de diciembre de 1.932, fol. 245; El Liberal, 12 de enero de 1.932, p. 2 y A.D.P.S., Leg. 550, Telegrama enviado a Hermenegildo Casas con el siguiente texto: "Causa mal tiempo suspéndese hasta domingo próximo homenaje bandera andaluza. salúdale, germa".

54 A.H.CA., Actas de la Diputación de Cádiz, sesión del 26 de enero de 1.933, fol. 17 .

55 De nuevo se nombran a Juan Antonio Campuzano y Miguel Maldonado como representantes a Córdoba. $\underline{\mathrm{Id}}$.

56 DIAZ ARRIAZA, J., y RUIZ ROMERO, M., op.cit., p. 120.

57 Las cartas enviadas por estos ayuntamientos en Ibíd, pp. 88-90.

58 HERCUES "Andalucismo. Balance de 1.932", Diario de Cádiz, 10 de enero de 1.932 .

\section{ABREVIATURAS:}

A.D.P.S.: Archivo de la Diputación Provincial de Sevilla

A.H.CA.: Archivo Histórico de Cádiz

A.M.CA.: Archivo Municipal de Cádiz. 